\title{
An existence theorem for a non-autonomous second order nonlocal multivalued problem
}

\author{
Tiziana Cardinali and Serena Gentili
}

\begin{abstract}
In this paper we prove the existence of mild solutions for a nonlocal problem governed by an abstract semilinear non-autonomous second order differential inclusion, where the non-linear part is an upper-Caratheodory semicontinuous multimap. Our existence theorem is obtained thanks to the introduction of a fundamental Cauchy operator. Finally we apply our main result to provide the controllability of a problem involving a non-autonomous wave equation.
\end{abstract}

Mathematics Subject Classification (2010): 34A60, 34G25.

Keywords: Nonlocal conditions, semilinear non-autonomous second order differential inclusion, fundamental Cauchy operator, fundamental system.

\section{Introduction}

Recently in [8] H.R. Henríquez, V. Poblete, J.C. Pozo have studied the existence of mild solutions for a nonlocal problem governed by the following non-autonomous wave equation

$$
\frac{\partial^{2} w(t, \xi)}{\partial t^{2}}=\frac{\partial^{2} w(t, \xi)}{\partial \xi^{2}}+b(t) \frac{\partial w(t, \xi)}{\partial \xi}+\tilde{f}(t, w(t, \xi)), t \in J=[0, a],
$$

Starting from this paper, we are interested to study the following control problem

$$
\left\{\begin{array}{l}
\frac{\partial^{2} w(t, \xi)}{\partial t^{2}}=\frac{\partial^{2} w(t, \xi)}{\partial \xi^{2}}+b(t) \frac{\partial w(t, \xi)}{\partial \xi}+f(t, w(t, \xi), u(t, \xi)), t \in J \\
w(t, 0)=w(t, 2 \pi), t \in J \\
\frac{\partial w(t, 0)}{\partial \xi}=\frac{\partial w(t, 2 \pi)}{\partial \xi}, t \in J \\
w(0, \xi)=\sum_{i=1}^{m} \frac{2 \pi t_{i}}{\xi_{i}}, \xi \in R \\
\frac{\partial w(0, \xi)}{\partial t}=\sum_{i=1}^{m} \frac{2 \pi}{\xi_{i}}, \xi \in R \\
u(t, \xi) \in U(t, w(t, \xi))
\end{array}\right.
$$

where $0<t_{1}<\ldots<t_{i}<\ldots<t_{m}<a$ and $0<\xi_{1}<\ldots<\xi_{i}<\ldots<\xi_{m}<2 \pi$, $b: J \rightarrow R, f: J \times R \times R \rightarrow R$ and $U: J \times R \rightarrow \mathcal{P}(R)$. 
By using the classical arguments (see, for example [15]) the controllability of (5.1) is brought back to the existence of mild solutions for a problem described by the nonautonomous semilinear second order differential inclusion with nonlocal conditions

$$
\left\{\begin{array}{l}
x^{\prime \prime}(t) \in A(t) x(t)+F(t, x(t)), t \in J \\
x(0)=g(x) \\
x^{\prime}(0)=h(x)
\end{array}\right.
$$

where $J=[0, a]$ is an interval of the real line, $\{A(t)\}_{t \in J}$ is a family of bounded, linear operators defined in a subspace $D(A(t))=D(A)$ dense in a real Banach space $X$ generating a "fundamental system", and $g, h$ are two operators defined on the trajectories and assuming values in $X$.

Recently the existence of nonlocal mild solutions in Banach space has been investigated for semilinear non-autonomous second order differential equations in [9], in [7] and in [8], while there exists an extensive literature for the autonomous case (see, for example, [6], [11], [12], [13] and [14]).

The note is organized in the following way. We start by introducing the fundamental Cauchy operator and by characterizing some of its properties, which play a key role to prove the existence of mild nonlocal solutions for problem (1.3) in the case that the nonlinear part of the semilinear second order differential inclusion is given by an upperCaratheodory semicontinuous multimap. In order to obtain our main existence result we use the powerful tools introduced in [9], [7] and a fixed point theorem for condensing multimaps. Our existence theorem extends in a broad sense all the existence results above mentioned. In the last section we apply our existence proposition for (1.3) in order to establish the controllability of (1.2).

\section{Preliminaries}

Let $X, Y$ be topological spaces and $\mathcal{P}(Y)$ be the family of all nonempty subsets of $Y$. We recall that a map $F: X \rightarrow \mathcal{P}(Y)$ is said to be upper semicontinuous (lower semicontinuous) if $F^{+}(V)=\{x \in X: F(x) \subset V\}\left(F^{-}(V)=\{x \in X\right.$ : $F(x) \cap V \neq \emptyset\})$ is an open subset of $X$, for every open $V \subset Y$; the multimap $F$ is said to have closed graph if the set graphF $=\{(x, y) \in X \times Y: y \in F(x)\}$ is closed in $X \times Y($ see $[5])$.

In this paper $X$ is a real Banach space endowed with a norm $\|\cdot\|$, and we will use the following notations:

$$
\begin{gathered}
\mathcal{P}_{b}(X)=\{H \in \mathcal{P}(X): H \text { bounded }\} \\
\mathcal{P}_{c}(X)=\{H \in \mathcal{P}(X): H \text { convex }\} ; \\
\mathcal{P}_{f}(X)=\{H \in \mathcal{P}(X): H \text { closed }\} ; \\
\mathcal{P}_{k}(X)=\{H \in \mathcal{P}(X): H \text { compact }\} ; \\
\mathcal{P}_{f c}(X)=\mathcal{P}_{f}(X) \cap \mathcal{P}_{c}(X) \ldots
\end{gathered}
$$

Further, let $J=[0, a]$ be an interval of the real line endowed with the usual Lebesgue measure $\lambda$.

A function $f: J \rightarrow X$ is said to be strongly measurable if there is a sequence of simple functions $\left(s_{n}\right)_{n}$ which converges to $f$ almost everywhere. 
Moreover, we denote by $C(J ; X)$ the space consisting of all continuous functions from $J$ into $X$ provided with the norm $\|.\|_{\infty}$ of uniform convergence, by $L^{1}(J, X)$ the space of all $X$-valued Bochner integrable functions on $J$ with norm $\|u\|_{1}=\int_{0}^{a}\|u(t)\| d t$ and $L_{+}^{1}(J)=\left\{f \in L^{1}(J, \mathbb{R}): f(t) \geq 0\right.$, for a.e. $\left.t \in J\right\}$.

A countable set $\left\{f_{n}\right\}_{n} \subset L^{1}(J, X)$ is said to be semicompact if: (i) $\left\{f_{n}\right\}_{n}$ is integrably bounded, i.e. there exists $\omega \in L_{+}^{1}(J)$ such that $\left\|f_{n}(t)\right\| \leq \omega(t)$, for a.e. $t \in J$ and for every $n \in \mathbb{N}$; (ii) the set $\left\{f_{n}(t)\right\}_{n}$ is relatively compact in $X$, for a.e. $t \in J$.

Now let us consider the following nonlocal problem governed by a non-autonomous abstract semilinear second order differential inclusion

$$
\left\{\begin{array}{l}
x^{\prime \prime}(t) \in A(t) x(t)+F(t, x(t)), t \in J \\
x(0)=g(x) \\
x^{\prime}(0)=h(x) .
\end{array}\right.
$$

In this problem $F$ is an $X$-valued multimap defined on $J \times X, g, h: C(J ; X) \rightarrow X$ are functions; $\{A(t)\}_{t \in J}$ is a family, generating a "fundamental system" $\{S(t, s)\}_{t, s \in J}$ of bounded linear operators $A(t): D(A) \rightarrow X$ (where $D(A)$ is a dense subspace of $X)$ such that, for each $x \in D(A)$, the function $t \mapsto A(t) x$ is continuous in $J$.

First we recall the concept of the "fundamental system", introduced by Kozak in [9] and recently used by H.R. Henríquez, V. Poblete, J.C. Pozo in [8].

Definition 2.1. A family $\{S(t, s)\}_{t, s \in J}$ of bounded linear operators $S(t, s): X \rightarrow X$ is called fundamental system generated by the family $\{A(t)\}_{t \in J}$ if (S1) for each $x \in X$, the function $S(.,) x:. J \times J \rightarrow X$ is of class $C^{1}$ and

(a) for each $t \in J, S(t, t) x=0, \forall x \in X$;

(b) for each $t, s \in J$ and for each $x \in X,\left.\frac{\partial}{\partial t} S(t, s)\right|_{t=s} x=x$ and

$$
\left.\frac{\partial}{\partial s} S(t, s)\right|_{t=s} x=-x
$$

(S2) for all $t, s \in J$, if $x \in D(A)$, then $S(t, s) x \in D(A)$, the map $S(.,) x:. J \times J \rightarrow X$ is of class $C^{2}$ and

(a) $\frac{\partial^{2}}{\partial t^{2}} S(t, s) x=A(t) S(t, s) x, \forall(t, s) \in J \times J, \forall x \in D(A)$;

(b) $\frac{\partial^{2}}{\partial s^{2}} S(t, s) x=S(t, s) A(s) x, \forall(t, s) \in J \times J, \forall x \in D(A)$;

(c) $\left.\frac{\partial^{2}}{\partial s \partial t} S(t, s)\right|_{t=s} x=0, \forall s \in J, \forall x \in D(A)$;

(S3) for all $s, t \in J$, if $x \in D(A)$, then $\frac{\partial}{\partial s} S(t, s) x \in D(A)$. Moreover, there exist $\frac{\partial^{3}}{\partial t^{2} \partial s} S(t, s) x$ and $\frac{\partial^{3}}{\partial s^{2} \partial t} S(t, s) x$ and

(a) $\frac{\partial^{3}}{\partial t^{2} \partial s} S(t, s) x=A(t) \frac{\partial}{\partial s} S(t, s) x, \forall(t, s) \in J \times J, \forall x \in D(A)$;

(b) $\frac{\partial^{3}}{\partial s^{2} \partial t} S(t, s) x=\frac{\partial}{\partial t} S(t, s) A(s) x, \forall(t, s) \in J \times J, \forall x \in D(A)$; and for all $x \in D(A)$ the function $(t, s) \mapsto A(t) \frac{\partial}{\partial s} S(t, s) x$ is continuous in $J \times J$.

Moreover, as in [8], a map $S: J \times J \rightarrow \mathcal{L}(X)$, where $\mathcal{L}(X)$ denote the space of all bounded linear operators in $X$ with the norm $\|\cdot\|_{\mathcal{L}(X)}$, is said to be a "fundamental operator" if $\{S(t, s)\}_{t, s \in J}$ is a fundamental system. Moreover, as in [8], we introduce, for each $(t, s) \in J \times J$ the operator

$$
C(t, s)=-\frac{\partial}{\partial s} S(t, s): X \rightarrow X
$$


By using the Banach-Steinhaus Theorem it is possible to prove that the fundamental system $\{S(t, s)\}_{t, s \in J}$ satisfies the following properties:

there exists two constants $K, K^{*}, K_{1}>0$ such that

$(p 1)\|C(t, s)\|_{\mathcal{L}(X)} \leq K, \forall(t, s) \in J \times J$

$(p 2)\|S(t, s)\|_{\mathcal{L}(X)} \leq K|t-s|, \forall t, s \in J$

$(p 3)\|S(t, s)\|_{\mathcal{L}(X)} \leq K a, \forall t, s \in J$

$(p 4)\left\|S\left(t_{2}, s\right)-S\left(t_{1}, s\right)\right\|_{\mathcal{L}(X)} \leq K^{*}\left|t_{2}-t_{1}\right|, \forall t_{1}, t_{2}, s \in J$

(p5) $\exists K_{1}>0:\left\|\frac{\partial}{\partial s} S\left(t_{2}, s\right)-\frac{\partial}{\partial s} S\left(t_{1}, s\right)\right\|_{\mathcal{L}(X)} \leq K_{1}\left|t_{2}-t_{1}\right|, \forall t_{1}, t_{2}, s \in J$.

Now we recall the definition of a mild solutions for the nonlocal problem (2.6)

Definition 2.2. A continuous function $u: J \rightarrow X$ is a mild solution for (2.6) if

$$
u(t)=-\left.\frac{\partial}{\partial s} S(t, s)\right|_{s=0} g(u)+S(t, 0) h(u)+\int_{0}^{t} S(t, \xi) f(\xi) d \xi, \forall t \in J
$$

where $f \in S_{F(., u(.))}^{1}=\left\{f \in L^{1}(J ; X): f(t) \in F(t, u(t))\right.$ a.e. $\left.t \in J\right\}$.

In the sequel let us denote by $0_{n}$ the zero-element of $\mathbb{R}^{n}$ and by $\preccurlyeq$ the partial ordering given by the standard positive cone $\mathbb{R}_{0,+}^{n}:=\left(\mathbb{R}_{0}^{+}\right)^{n}$, i.e. $x \preccurlyeq y$ if and only if $y-x \in \mathbb{R}_{0,+}^{n}$; clearly, $x \prec y$ means that $x \preccurlyeq y$ and $x \neq y$.

Definition 2.3. A function $\beta: \mathcal{P}_{b}(X) \rightarrow \mathbb{R}_{0,+}^{n}$ is said to be a "measure of noncompactness" (MNC, for short) in the Banach space $X$ if, for every $\Omega \in \mathcal{P}_{b}(X)$, the following properties are satisfied:

$\left(\beta_{1}\right) \beta(\Omega)=0_{n}$ if and only if $\bar{\Omega}$ is compact;

$\left(\beta_{2}\right) \beta(\overline{c o}(\Omega))=\beta(\Omega)$.

Further, a MNC $\beta: \mathcal{P}_{b}(X) \rightarrow \mathbb{R}_{0,+}^{n}$ is said to be:

monotone if $\Omega_{1}, \Omega_{2} \in \mathcal{P}_{b}(X): \Omega_{1} \subset \Omega_{2}$ implies $\beta\left(\Omega_{1}\right) \preccurlyeq \beta\left(\Omega_{2}\right)$;

nonsingular if $\beta(\{x\} \cup \Omega)=\beta(\Omega)$, for every $x \in X, \Omega \in \mathcal{P}_{b}(X)$;

invariant under closure if $\beta(\bar{\Omega})=\beta(\Omega), \Omega \in \mathcal{P}_{b}(X)$;

invariant with respect to the union with compact set if $\beta(\Omega \cup C)=\beta(\Omega)$, for every relatively compact set $C \subset X$ and $\Omega \in \mathcal{P}_{b}(X)$.

In this setting we provide the following definitions (see [2]).

Definition 2.4. If $D$ is a nonempty subset of $X$, a map $\phi: D \rightarrow \mathcal{P}(X)$ is said to be "condensing" with respect to a MNC $\beta: \mathcal{P}_{b}(X) \rightarrow \mathbb{R}_{0,+}^{n}$ (shortly, " $\beta$-condensing ") if (I) $\phi(D)$ is bounded

and

(II) $\beta(\Omega) \preccurlyeq \beta(\phi(\Omega))$ implies $\beta(\Omega)=0_{n}, \Omega \in \mathcal{P}_{b}(D)$

or equivalently

(II)' $0_{n} \prec \beta(\Omega)$ implies $\beta(\Omega) \npreceq \beta(\phi(\Omega)), \Omega \in \mathcal{P}_{b}(D)$ (i.e. $\beta(\phi(\Omega)) \prec \beta(\Omega)$ is true or $\beta(\phi(\Omega))$ and $\beta(\Omega)$ are not comparable).

We can now recall the following Sadowskii type fixed point theorem for multimaps condensing with respect to a vectorial measure of noncompactness (see [2], Theorem $2.2)$. 
Theorem 2.5. Let $\beta: \mathcal{P}_{b}(X) \rightarrow \mathbb{R}_{0,+}^{n}$ be a nonsingular $M N C, D$ be a closed convex subset of a Banach space $X$ and $\phi: D \rightarrow \mathcal{P}_{f c}(D)$ be a map such that

$(\phi 1) \phi$ has weakly closed graph in $D \times X$, i.e. for every sequence $\left(x_{n}\right)_{n}$ in $D, x_{n} \rightarrow x$, $x \in D$, and for every sequence $\left(y_{n}\right)_{n}, y_{n} \in \phi\left(x_{n}\right), y_{n} \rightarrow y$, then $S(x, y) \cap \phi(x) \neq 0$, where $S(x, y)=\{x+\lambda(y-x): \lambda \in[0,1]\}$;

( $\phi 2) \phi$ is $\beta$-condensing.

Then there exists $x \in D$ with $x \in \phi(x)$.

Next, we consider the set $\mathbb{R}_{0,+}^{2}=\mathbb{R}_{0}^{+} \times \mathbb{R}_{0}^{+}$endowed with the partial ordering $\preccurlyeq$ before introduced. Fixed a constant $L \geq 0$ we can introduce the function $\nu_{L}: \mathcal{P}_{b}(C(J ; X)) \rightarrow$ $\mathbb{R}_{0,+}^{2}$ defined by

$$
\nu_{L}(\Omega)=\max _{\left\{w_{n}\right\}_{n} \subset \Omega}\left(\tau\left(\left\{w_{n}\right\}_{n}\right), \lambda\left(\left\{w_{n}\right\}_{n}\right)\right), \forall \Omega \in \mathcal{P}_{b}(C(J ; X)),
$$

being

$$
\tau\left(\left\{w_{n}\right\}_{n}\right)=\sup _{t \in J} e^{-L t} \eta\left(\left\{w_{n}(t)\right\}_{n}\right)
$$

and

$$
\lambda\left(\left\{w_{n}\right\}_{n}\right)=\bmod _{C}\left(\left\{w_{n}\right\}_{n}\right)
$$

where $\eta$ is the Hausdorff MNC in the Banach space $X$ and $\bmod _{C}$ is the modulus of continuity in $C(J ; X)$ (see $[3])$.

\section{The fundamental Cauchy operator}

To study the problem (2.6) we introduce the following operator, which will play a key role in our next existence result.

Definition 3.1. Let $\{S(t, s)\}_{t, s \in J}$ be the fundamental system generated by the family $\{A(t)\}_{t \in J}$ of bounded linear operators in the Banach space $X$, presented in (2.6). We will call the operator $G_{S}: L^{1}(J ; X) \rightarrow C(J ; X)$ defined by

$$
G_{S} f(t)=\int_{0}^{t} S(t, s) f(s) d s, t \in J=[0, a], f \in L^{1}(J ; X)
$$

the fundamental Cauchy operator.

First we present the following result, which is analogous to the one proved in [10] or in [4], respectively for the Cauchy operator and for the generalized Cauchy operator.

Theorem 3.2. The fundamental Cauchy operator $G_{S}$ satisfies the following properties: $\left(G_{S} 1\right)\left\|G_{S} f(t)-G_{S} g(t)\right\| \leq K a \int_{0}^{t}\|f(s)-g(s)\| d s, t \in J, f, g \in L^{1}(J ; X) ;$

where $K a$ is the constant presented in $(p 3)$;

$\left(G_{S} 2\right)$ for any compact $H \subset X$ and sequence $\left(f_{n}\right)_{n}, f_{n} \in L^{1}(J ; X)$, such that $\left\{f_{n}(t)\right\}_{n} \subset H$ for a.e. $t \in J$, the weak convergence $f_{n} \rightarrow f_{0}$ implies the convergence $G_{S} f_{n} \rightarrow G_{S} f_{0}$. 
Proof. Let $t \in J$ and $f, g \in L^{1}(J ; X)$ be fixed. Thanks to the definition of the fundamental Cauchy operator and to the property $(p 3)$ we have:

$$
\left\|G_{S} f(t)-G_{S} g(t)\right\|=\left\|\int_{0}^{t} S(t, s)(f(s)-g(s)) d s\right\| \leq K a \int_{0}^{t}\|f(s)-g(s)\| d s
$$

Therefore we can deduce that $G_{S}$ satisfies $\left(G_{S} 1\right)$.

Let us prove the property $\left(G_{S} 2\right)$.

Fix a compact set $H \subset X$ and $t \in J$, let us consider the set $Q_{t} \subseteq X$ defined as folllows:

$$
Q_{t}=\bigcup_{s \in[0, t]} S(t, s) H
$$

Now we show that $Q_{t}$ is compact. Let us fix the map $q_{H}: J \times J \times H \rightarrow X$ defined by $q_{H}(t, s, x)=S(t, s) x,(t, s, x) \in J \times J \times H$. Then, for each $\varepsilon>0$, there exist finitely many $x_{1}, \ldots, x_{p} \in X$ such that:

$$
H \subset \cup_{i=1}^{p}\left(x_{i}+\frac{\varepsilon}{4 K a} B_{1}(0)\right)
$$

where $B_{1}(0)$ is the open unit ball in $X$.

Now, by the strongly continuity of $S$, there exists $\eta_{H}(\varepsilon)>0$ such that for every $\left(t_{1}, s_{1}\right),\left(t_{2}, s_{2}\right) \in \Delta$ with $\max \left\{\left|t_{1}-t_{2}\right|,\left|s_{1}-s_{2}\right|\right\}<\eta_{H}(\varepsilon)$ we have

$$
\left\|S\left(t_{1}, s_{1}\right) x_{i}-S\left(t_{2}, s_{2}\right) x_{i}\right\|<\frac{\varepsilon}{4}, \text { for every } i=1, \ldots, p .
$$

Put $\tilde{\eta}_{H}(\varepsilon)=\min \left\{\eta_{H}(\varepsilon), \frac{\varepsilon}{4 K a}\right\}$, for arbitrary $\left(t_{1}, s_{1}, z_{1}\right),\left(t_{2}, s_{2}, z_{2}\right) \in J \times J \times H$ such that $\max \left\{\left|t_{1}-t_{2}\right|,\left|s_{1}-s_{2}\right|,\left\|z_{1}-z_{2}\right\|\right\}<\tilde{\eta}_{H}(\varepsilon)$, since there exists $j \in\{1, \ldots, p\}$ such that $\left\|z_{1}-x_{j}\right\|<\frac{\varepsilon}{4 K a}$, by $(p 3)$ and (3.4) we get

$$
\begin{aligned}
& \left\|q_{H}\left(t_{1}, s_{1}, z_{1}\right)-q_{H}\left(t_{2}, s_{2}, z_{2}\right)\right\|=\left\|S\left(t_{1}, s_{1}\right) z_{1}-S\left(t_{2}, s_{2}\right) z_{2}\right\| \\
\leq & \left\|S\left(t_{1}, s_{1}\right) z_{1}-S\left(t_{1}, s_{1}\right) x_{j}\right\|+\left\|S\left(t_{1}, s_{1}\right) x_{j}-S\left(t_{2}, s_{2}\right) x_{j}\right\| \\
+ & \left\|S\left(t_{2}, s_{2}\right) x_{j}-S\left(t_{2}, s_{2}\right) z_{1}\right\|+\left\|S\left(t_{2}, s_{2}\right) z_{1}-S\left(t_{2}, s_{2}\right) z_{2}\right\| \\
\leq & \left\|S\left(t_{1}, s_{1}\right)\left(z_{1}-x_{j}\right)\right\|+\left\|\left[S\left(t_{1}, s_{1}\right)-S\left(t_{2}, s_{2}\right)\right] x_{j}\right\| \\
+ & \left\|S\left(t_{2}, s_{2}\right)\left(x_{j}-z_{1}\right)\right\|+\left\|S\left(t_{2}, s_{2}\right)\left(z_{1}-z_{2}\right)\right\| \\
\leq & K a\left\|z_{1}-x_{j}\right\|+\frac{\varepsilon}{4}+K a\left\|x_{j}-z_{1}\right\|+K a\left\|z_{1}-z_{2}\right\|<\varepsilon .
\end{aligned}
$$

Therefore the map $q_{H}$ is uniformly continuous. Hence, being true that

$$
Q_{t}=q_{H}(\{t\} \times[0, t] \times H),
$$

we can say that $Q_{t}$ is compact.

Now, we show that, for every sequence $\left(f_{n}\right)_{n}, f_{n} \in L^{1}(J ; X)$, such that $\left\{f_{n}(t)\right\}_{n} \subset H$ a.e. $t \in J$ we have that the set $\left\{G_{S} f_{n}(t)\right\}_{n}$ is relatively compact in $X$, for every $t \in J$. To this end, fixed $t \in J$, it is enough to prove that

$$
\left\{G_{S} f_{n}(t)\right\}_{n} \subset t \overline{c o}\left(Q_{t}\right) .
$$


Let us associate to $\left(f_{n}\right)_{n=1}^{+\infty}$ a sequence $\left(\tilde{f}_{n}\right)_{n=1}^{+\infty}$ such that $\tilde{f}_{n}(t) \in H$ for every $t \in J$ and $\tilde{f}_{n}=f_{n}$ a.e. in $J$. By applying [5, Corollary 3.10.19], we obtain

$$
G_{S} f_{n}(t)=\int_{0}^{t} S(t, s) \tilde{f}_{n}(s) d s \in t \overline{c o}\left\{S(t, s) \tilde{f}_{n}(s): s \in[0, t]\right\} \subset t \overline{c o} Q_{t}, \forall n \in \mathbb{N} .
$$

Next, let us show now that $\left\{G_{S} f_{n}\right\}_{n=1}^{+\infty} \subset C(J ; X)$ is (uniformly) equicontinuous in $J$. To see this, fixed $\varepsilon>0$, we can choose $\delta(\varepsilon)=\frac{\varepsilon}{a K_{2}\left(K+K^{*}\right)}$, where the constants $K$ and $K^{*}$ are respectively that from properties $(p 1)$ and $(p 4)$ in Section 2 , while $K_{2}$ is a constant such that

$$
\left\|f_{n}(t)\right\| \leq K_{2} \text {, a.e. } t \in J, \forall n \in \mathbb{N} .
$$

Then, for every $t_{1}, t_{2} \in J,\left|t_{2}-t_{1}\right| \leq \delta(\varepsilon)$, and w.l.o.g. $t_{2}>t_{1}$, we have (see $(p 3)$ and (p4) in Section 2):

$$
\begin{gathered}
\left\|G_{S} f_{n}\left(t_{2}\right)-G_{S} f_{n}\left(t_{1}\right)\right\| \leq \int_{0}^{t_{1}}\left\|\left[S\left(t_{2}, s\right)-S\left(t_{1}, s\right)\right] f_{n}(s)\right\| d s \\
+\int_{t_{1}}^{t_{2}}\left\|S\left(t_{2}, s\right)\right\|_{\mathcal{L}(X)}\left\|f_{n}(s)\right\| d s \\
\leq \int_{0}^{t_{1}}\left\|S\left(t_{2}, s\right)-S\left(t_{1}, s\right)\right\|_{\mathcal{L}(X)}\left\|f_{n}(s)\right\| d s+K_{2} K a\left(t_{2}-t_{1}\right) \\
\leq \int_{0}^{a} K^{*}\left(t_{2}-t_{1}\right) K_{2} d s+K_{2} K a\left(t_{2}-t_{1}\right) \leq \delta(\varepsilon) a K_{2}\left(K+K^{*}\right)=\varepsilon .
\end{gathered}
$$

Hence we have the equicontinuity in $J$ of the set $\left\{G_{S} f_{n}\right\}_{n}$.

Furthermore, the condition $\left(G_{S} 1\right)$ implies that the linear operator $G_{S}$ is bounded, hence $G_{S}$ is weakly continuous, i.e. if $f_{n} \rightarrow f_{0}$ then $G_{S} f_{n} \rightarrow G_{S} f_{0}$. Now, by applying a generalized version of the Ascoli-Arzelà criterion obtained by Ambrosetti in [1], we get the relative compactness of the set $\left\{G_{S} f_{n}\right\}_{n}$.

The relative compactness of $\left\{G_{S} f_{n}\right\}_{n=1}^{+\infty}$ provides that the last convergence is in the norm of the space $C(J ; X)$. So also $\left(G_{S} 2\right)$ is stated.

Remark 3.3. Condition $\left(G_{S} 1\right)$ obviously implies the Lipschitz condition $\left(G_{S} 1\right)^{\prime}\left\|G_{S} f-G_{S} g\right\|_{\infty} \leq K a\|f-g\|_{1}$, for all $f, g \in L^{1}(J ; X)$.

\section{Existence results}

Thanks to the properties of the fundamental Cauchy operator we are able to prove our main existence result.

Theorem 4.1. Let $J=[0, a], X$ a Banach space and $\{A(t)\}_{t \in J}$ a family which satisfies the property:

(A) $\{A(t)\}_{t \in J}$ is a family of bounded linear operators, defined in a subspace $D(A)$ dense in $X$ and taking values in $X$, generating a fundamental system $\{S(t, s)\}_{(t, s) \in J \times J}$ such that, for each $x \in D(A)$, the function $t \mapsto A(t) x$ is continuous in $J$.

Let $F: J \times X \rightarrow \mathcal{P}_{k c}(X)$ a multimap which satisfies the following hypothesis:

(F1) for every $x \in X, F(., x)$ admits a B-measurable selector;

(F2) for a.e. $t \in J, F(t,$.$) is upper semicontinuous;$ 
(F3) there exists a function $\alpha \in L_{+}^{1}(J)$ such that

$$
\|F(t, x)\|=\sup _{z \in F(t, x)}\|z\| \leq \alpha(t)(1+\|x\|)
$$

for a.e. $t \in J$ and for all $x \in X$.

(F4) there exists a function $m \in L_{+}^{1}(J)$ such that

$$
\eta(F(t, B)) \leq m(t) \eta(B)
$$

for a.e. $t \in J$ and for every $B \in \mathcal{P}_{b}(X)$ (where $\eta$ is the Hausdorff $M N C$ in $X$ ).

Let $g, h: C(J ; X) \rightarrow X$ be two functions which satisfy the following properties:

(gh1) $g, h$ are compact, i.e. they are continuous and map bounded sets into relatively compact sets;

(gh2) there exists $Q>0:\|g(u)\| \leq Q,\|h(u)\| \leq Q$ for every $u \in C(J ; X)$.

Then there exists at least one mild solution for the nonlocal problem (2.6).

Proof. We consider the integral multioperator $\Gamma: C(J ; X) \rightarrow \mathcal{P}_{c}(C(J ; X))$ defined as

$$
\begin{aligned}
\Gamma(u)= & \{y \in C(J ; X): y(t)=C(t, 0) g(u)+S(t, 0) h(u) \\
& \left.+\int_{0}^{t} S(t, \xi) f(\xi) d \xi, t \in J, f \in S_{F(., u(.))}^{1}\right\}
\end{aligned}
$$

for all $u \in C(J ; X)$.

Note that, for all $u \in C(J ; X)$, since $S_{F(., u(.))} \neq \emptyset$ (see [10], Lemma 5.1.1) we have $\Gamma(u) \neq \emptyset$. Moreover $\Gamma$ takes convex values thanks the convexity of the values of $F$. From now on we proceed by steps.

Step 1: There exists a set which is invariant under the action of the operator $\Gamma$.

Step 1a: We put

$$
q_{n}=\max _{t \in J}\left\{\int_{0}^{t} K a e^{-n(t-s)} \alpha(s) d s\right\}
$$

for all $n \in \mathbb{N}$, where $K, \alpha$ are respectively from $(p 1)$ and $(F 3)$ and $a$ is the size of $J$. Let us show that

$$
\inf _{n \in \mathbb{N}} q_{n}=0 .
$$

From definition of maximum, for all $n \in \mathbb{N}$, there exists $t_{n} \in J$ such that

$$
q_{n}-\frac{1}{n}<\int_{0}^{t_{n}} K a e^{-n\left(t_{n}-s\right)} \alpha(s) d s=\int_{0}^{a} \psi_{n}(s) d s
$$

being $\psi_{n}: J \rightarrow \mathbb{R}$ the function defined as follows

$$
\psi_{n}(s)=K a e^{-n\left(t_{n}-s\right)} \chi_{\left[0, t_{n}\right]}(s) \alpha(s) \text {, for all } s \in J,
$$

where $\chi_{\left[0, t_{n}\right]}$ is the characteristic function of the interval $\left[0, t_{n}\right]$. Eventually passing to a subsequence, the sequence $\left(\psi_{n}\right)_{n}$ is such that

$$
\begin{gathered}
\lim _{n \rightarrow \infty} \psi_{n}(s)=0, \text { for all } s \in J \\
\left|\psi_{n}(s)\right| \leq K a \alpha(s)=: \alpha^{*}(s), \forall s \in J, \forall n \in \mathbb{N},
\end{gathered}
$$

where $\alpha^{*} \in L_{+}^{1}(J)$. So the Dominated Convergence Theorem implies that

$$
\lim _{n \rightarrow \infty} \int_{0}^{a} \psi_{n}(s) d s=0 .
$$


Since $q_{n} \geq 0$, by (4.4) we obtain $\lim _{n \rightarrow \infty} q_{n}=0$. Hence (4.3) is proved.

Therefore, there exists $N \in \mathbb{N}$ such that

$$
q_{N}<1
$$

Now, let us consider the norm $\|\cdot\|_{N}: C(J ; X) \rightarrow \mathbb{R}_{0}^{+}$defined by:

$$
\|u\|_{N}=\max _{t \in J} e^{-N t}\|u(t)\|, \forall u \in C(J ; X),
$$

which is equivalent to the usual norm $\|\cdot\|_{\infty}$ in $C(J ; X)$ (cfr. [10], (5.2.7)).

Let us fix

$$
R \geq \frac{K\left(Q+a Q+a\|\alpha\|_{1}\right)}{1-q_{N}}
$$

where $K$ is the constant in $(p 1), a$ is the size of the interval $J, Q$ and $q_{N}$ are respectively from $(g h 2)$ and (4.5).

Step 1b: Now, we consider (see (4.7))

$$
H_{R}=\left\{u \in C(J ; X):\|u\|_{N} \leq R\right\},
$$

the closed ball in $\left(C(J ; X),\|\cdot\|_{N}\right)$.

We show that

$$
\Gamma\left(H_{R}\right) \subset H_{R}
$$

Fixed $u \in H_{R}$ and $y \in \Gamma(u)$, for every $t \in J$, we have

$$
\begin{aligned}
e^{-N t}\|y(t)\| \leq & e^{-N t}\|C(t, 0) g(u)\|+e^{-N t}\|S(t, 0) h(u)\|+ \\
& +e^{-N t} \int_{0}^{t}\|S(t, \xi) f(\xi)\| d \xi
\end{aligned}
$$

Then by using $(p 1),(p 2),(F 3),(g h 2)$ and (4.6) we have

$$
\begin{gathered}
e^{-N t}\|y(t)\| \leq\|C(t, 0)\|_{\mathcal{L}(X)}\|g(u)\|+\|S(t, 0)\|_{\mathcal{L}(X)}\|h(u)\|+ \\
+e^{-N t} \int_{0}^{t}\|S(t, \xi)\|_{\mathcal{L}(X)}\|f(\xi)\| d \xi \leq \\
\leq K\left(Q+a Q+a\|\alpha\|_{1}\right)+e^{-N t} K a \int_{0}^{t} \alpha(\xi)\|u(\xi)\| d \xi= \\
=K\left(Q+a Q+a\|\alpha\|_{1}\right)+e^{-N t} K a \int_{0}^{t} e^{N \xi} \alpha(\xi) e^{-N \xi}\|u(\xi)\| d \xi \leq \\
\leq K\left(Q+a Q+a\|\alpha\|_{1}\right)+\|u\|_{N} K a \int_{0}^{t} e^{-N(t-\xi)} \alpha(\xi) d \xi .
\end{gathered}
$$

So, recalling that $u \in H_{R}$, by (4.8), (4.7) and ((4.2) for $\left.\mathrm{n}=\mathrm{N}\right)$ we obtain

$$
\begin{gathered}
e^{-N t}\|y(t)\| \leq K\left(Q+a Q+a\|\alpha\|_{1}\right)+R \int_{0}^{t} K a e^{-N(t-\xi)} \alpha(\xi) d \xi \leq \\
\leq K\left(Q+a Q+a\|\alpha\|_{1}\right)+R q_{N} \leq R .
\end{gathered}
$$

Now, by (4.6), we have

$$
\|y\|_{N} \leq R
$$

hence $y \in H_{R}$. Therefore (4.9) is true.

Step 2: In order to prove the existence of a mild solution for (2.6) it is enough to have 
the existence of a fixed point for the restriction $\Gamma_{\left.\right|_{H_{R}}}\left(\right.$ shortly $\left.\Gamma_{R}\right)$, i.e. (see (4.9)) for the map

$$
\Gamma_{R}: H_{R} \rightarrow \mathcal{P}_{c}\left(H_{R}\right)
$$

To this aim, we will show that $\Gamma_{R}$ satisfies all the hypotheses of Theorem 2.5, where the Banach space considered is $\left(C(J ; X),\|.\|_{\infty}\right)$ (shortly $\left.C(J ; X)\right)$. Obviously $H_{R}$, which is a closed ball in the space $\left(C(J ; X),\|.\|_{N}\right)$, is a closed and convex subset of $C(J ; X)$.

Step 2a: The integral multioperator $\Gamma_{R}$ has closed graph.

Let $\left(u_{n}\right)_{n}$ be a sequence in $H_{R}$ such that $u_{n} \rightarrow \bar{u}$ and let $\left(z_{n}\right)_{n}$ be a sequence such that $z_{n} \in \Gamma\left(u_{n}\right), \forall n \in \mathbb{N}$, and $z_{n} \rightarrow \bar{z}$ in $C(J ; X)$.

Moreover, let $\left(f_{n}\right)_{n}$ be a sequence such that, for every $n \in \mathbb{N}, f_{n} \in S_{F\left(., u_{n}(.)\right)}^{1}$, and

$$
z_{n}(t)=C(t, 0) g\left(u_{n}\right)+S(t, 0) h\left(u_{n}\right)+\int_{0}^{t} S(t, \xi) f_{n}(\xi) d \xi, \text { for all } t \in J
$$

Now, let us note that the set $\left\{f_{n}\right\}_{n}$ is integrably bounded. This follows from the boundness of the set $\left\{u_{n}\right\}_{n}$ in $C(J, X)$ and from $(F 3)$.

Furthermore, let us show that the set $\left\{f_{n}(t)\right\}_{n}$ is relatively compact in $X$ for a.e. $t \in J$. Indeed, by using $(F 4)$ and the monotonicity of the Hausdorff MNC, for a.e. $t \in J$, being $\left\{u_{n}(t)\right\}_{n} \in \mathcal{P}_{b}(X)$, we can write the extimate

$$
\eta\left(\left\{f_{n}(t)\right\}_{n}\right) \leq \eta\left(F\left(t,\left\{u_{n}(t)\right\}_{n}\right)\right) \leq m(t) \eta\left(\left\{u_{n}(t)\right\}_{n}\right) .
$$

Next, since the set $\left\{u_{n}(t)\right\}_{n}$ is relatively compact in $X$, from (4.12), we have $\eta\left(\left\{f_{n}(t)\right\}_{n}\right)=0$, i.e. the set $\left\{f_{n}(t)\right\}_{n}$ is relatively compact.

Now, we can use [[10], Proposition 4.2.1] to conclude that the set $\left\{f_{n}\right\}_{n}$ is weakly compact in $L^{1}(J ; X)$, so w.l.o.g. we can assume $f_{n} \rightarrow \bar{f}$ in $L^{1}(J ; X)$.

Then, in virtue of Theorem 3.2 and Remark 3.3 we can say that the fundamental Cauchy operator satisfies $\left(G_{S} 1\right)^{\prime}$ and $\left(G_{S} 2\right)$. Therefore, since the set $\left\{f_{n}\right\}_{n}$ is semicompact we can apply [[10], Theorem 5.1.1] and deduce

$$
G_{S} f_{n} \rightarrow G_{S} \bar{f} \text { in } C(J ; X) .
$$

Moreover, fixed $t \in J$, since $C(t, 0), S(t, 0) \in \mathcal{L}(X)$ and $g, h$ are continuous in $C(J ; X)$ (see hypothesis $(g h 1)$ ), we have:

$$
\begin{aligned}
& C(t, 0) g\left(u_{n}\right) \rightarrow C(t, 0) g(\bar{u}), \text { per } n \rightarrow \infty \\
& S(t, 0) h\left(u_{n}\right) \rightarrow S(t, 0) h(\bar{u}), \text { per } n \rightarrow \infty
\end{aligned}
$$

Hence, by passing to the limit in (4.11), from (4.13), (4.14)), (4.15) we have

$$
\begin{aligned}
\lim _{n \rightarrow \infty} z_{n}(t) & =\lim _{n \rightarrow \infty}\left[C(t, 0) g\left(u_{n}\right)+S(t, 0) h\left(u_{n}\right)+G_{S} f_{n}(t)\right]= \\
& =C(t, 0) g(\bar{u})+S(t, 0) h(\bar{u})+G_{S} \bar{f}(t)
\end{aligned}
$$

Now, the uniqueness of the limit algorithm guarantees that (see (3.1)):

$$
\bar{z}(t)=C(t, 0) g(\bar{u})+S(t, 0) h(\bar{u})+\int_{0}^{t} S(t, \xi) \bar{f}(\xi) d \xi, \quad \text { for every } t \in J .
$$


By [[10], Lemma 5.1.1], we have that $\bar{f} \in S_{F(., \bar{u}(.))}^{1}$, hence we can conclude that $\bar{z} \in \Gamma_{R}(\bar{u})$. Therefore, $\Gamma_{R}$ has closed graph.

Step 2b: For every $l \in \mathbb{N}$ we can consider the real number

$$
p_{l}:=\max _{t \in J} \int_{0}^{t} 2 K a e^{-l(t-s)} m(s) d s
$$

where $K, a, m$ are respectively from $(p 1),(2.6)$ and $(F 4)$. By means of similar arguments as the ones used to prove (4.5), we can choose $l=L$ large enough so that

$$
p_{L}<1 \text {. }
$$

In correspondence to such an $L$ we consider the vectorial MNC $\nu_{L}$ on $C(J ; X)$ defined in $(2.8)$.

Next, we prove that the integral multioperator $\Gamma_{R}$ is $\nu_{L}$-condensing.

First, by (4.9) the equivalence of the norms $\|\cdot\|_{\infty}$ and $\|\cdot\|_{N}$ implies the boundness of the set $\Gamma_{R}\left(H_{R}\right)$ in $\left(C(J ; X),\|\cdot\|_{\infty}\right)$ Therefore, condition $(I)$ of $\nu_{L}$-consensivity holds. Now we show that condition $(I I)$ is satisfied too. So let $\Omega \subset H_{R}$ be a bounded set such that

$$
\nu_{L}(\Omega) \preccurlyeq \nu_{L}\left(\Gamma_{R}(\Omega)\right),
$$

we will prove that $\nu_{L}(\Omega)=0_{2}$.

Recalling that $\nu_{L}\left(\Gamma_{R}(\Omega)\right.$ ) is a maximum (see (2.8)), we consider the countable set $\left\{y_{n}\right\}_{n} \subset \Gamma_{R}(\Omega)$ which achieves that maximum. Let now $\left\{u_{n}\right\}_{n} \subset \Omega$ be a set such that $y_{n} \in \Gamma_{R}\left(u_{n}\right), n \in \mathbb{N}$. Moreover, for every $n \in \mathbb{N}$, by (4.1), (4.10), (3.1) there exists $f_{n} \in S_{F\left(., u_{n}(.)\right)}^{1}$ such that

$$
y_{n}(t)=C(t, 0) g\left(u_{n}\right)+S(t, 0) h\left(u_{n}\right)+G_{S} f_{n}(t), t \in J .
$$

Of course, since (4.18) holds, we have (see (2.8))

$$
\left(\tau\left(\left\{u_{n}\right\}_{n}\right), \lambda\left(\left\{u_{n}\right\}_{n}\right)\right) \preccurlyeq \nu_{L}(\Omega) \preccurlyeq \nu_{L}\left(\Gamma_{R}(\Omega)\right)=\left(\tau\left(\left\{y_{n}\right\}_{n}\right), \lambda\left(\left\{y_{n}\right\}_{n}\right)\right) .
$$

First of all, from the above relation we have the inequality

$$
\tau\left(\left\{u_{n}\right\}_{n}\right) \leq \tau\left(\left\{y_{n}\right\}_{n}\right) .
$$

Let us estimate (cf. (2.9))

$$
\tau\left(\left\{y_{n}\right\}_{n}\right)=\sup _{t \in J} e^{-L t} \eta\left(\left\{y_{n}(t)\right\}_{n}\right) .
$$

Fixed $t \in J$, by using (4.19), $(p 1)$ and $(p 2)$ of the fundamental system and the properties of $\eta$, we have

$$
\begin{gathered}
\eta\left(\left\{y_{n}(t)\right\}_{n}\right) \leq \eta\left(\left\{C(t, 0) g\left(u_{n}\right)\right\}_{n}\right)+\eta\left(\left\{S(t, 0) h\left(u_{n}\right)\right\}_{n}\right)+\eta\left(\left\{G_{S} f_{n}(t)\right\}_{n}\right) \leq \\
\leq K \eta\left(g\left(\left\{u_{n}\right\}_{n}\right)\right)+K a \eta\left(h\left(\left\{u_{n}\right\}_{n}\right)\right)+\eta\left(\left\{G_{S} f_{n}(t)\right\}_{n}\right) .
\end{gathered}
$$

Being $\left\{u_{n}\right\}_{n}$ a bounded set, from hypothesis $(g h 1)$ we can deduce that $\eta\left(g\left(\left\{u_{n}\right\}\right)_{n}\right)=$ 0 and $\eta\left(h\left(\left\{u_{n}\right\}\right)_{n}\right)=0$. Therefore, by (4.23) we have

$$
\eta\left(\left\{y_{n}(t)\right\}_{n}\right) \leq \eta\left(\left\{G_{S} f_{n}(t)\right\}_{n}\right) .
$$


In order to apply Theorem 4.2.2 of [10], we first note that the boundness of $\left\{u_{n}\right\}_{n}$ in $C(J ; X)$ and $(F 3)$ imply that the set $\left\{f_{n}\right\}_{n}$ is integrably bounded. Moreover by $(F 4)$, for a.e. $s \in J$, we have

$$
\begin{gathered}
\\
\eta\left(\left\{f_{n}(s)\right\}_{n}\right) \leq \eta\left(F\left(s,\left\{u_{n}(s)\right\}_{n}\right)\right) \leq m(s) \eta\left(\left\{u_{n}(s)\right\}_{n}\right) \leq \\
\leq e^{L s} m(s) \sup _{\xi \in J} e^{-L \xi} \eta\left(\left\{u_{n}(\xi)\right\}_{n}\right)=e^{L s} m(s) \tau\left(\left\{u_{n}\right\}_{n}\right)=: v(s)
\end{gathered}
$$

where obviously $v \in L_{+}^{1}(J)$.

On the other hand, by using Theorem 3.2 we know that the fundamental Cauchy operator $G_{S}$ satisfies $\left(G_{S} 1\right)$ and $\left(G_{S} 2\right)$. Now we are in the position to apply Theorem 4.2 .2 of [10], so we get (cf. (4.25)):

$$
\eta\left(\left\{G_{S} f_{n}(t)\right\}_{n}\right) \leq 2 K a \int_{0}^{t} v(s) d s=2 K a \tau\left(\left\{u_{n}\right\}_{n}\right) \int_{0}^{t} e^{L s} m(s) d s, \forall t \in J,
$$

hence, by (4.24) and (4.26) we have

$$
\eta\left(\left\{y_{n}(t)\right\}_{n}\right) \leq 2 \operatorname{Ka\tau }\left(\left\{u_{n}\right\}_{n}\right) \int_{0}^{t} e^{L s} m(s) d s, \forall t \in J .
$$

From this last inequality, remembering (2.9) and (4.16) with $l=L$, we obtain

$$
\tau\left(\left\{y_{n}\right\}_{n}\right) \leq \sup _{t \in J}\left[2 K a \tau\left(\left\{u_{n}\right\}_{n}\right) \int_{0}^{t} e^{-L(t-s)} m(s) d s\right] \leq p_{L} \tau\left(\left\{u_{n}\right\}_{n}\right)
$$

Therefore (4.21) and (4.27) imply

$$
\tau\left(\left\{u_{n}\right\}_{n}\right) \leq \tau\left(\left\{y_{n}\right\}_{n}\right) \leq p_{L} \tau\left(\left\{u_{n}\right\}_{n}\right),
$$

and so, since $p_{L}<1$ (4.17), we achieve

$$
\tau\left(\left\{u_{n}\right\}_{n}\right)=0 .
$$

By (4.28) we also deduce

$$
\tau\left(\left\{y_{n}\right\}_{n}\right)=0
$$

Now we show that (cf. (2.10))

$$
\lambda\left(\left\{y_{n}\right\}_{n}\right)=\bmod _{C}\left(\left\{y_{n}\right\}_{n}\right)=0
$$

To this aim, we prove that $\bmod _{C}\left(\left\{y_{n}\right\}_{n}\right)=0$. Indeed, from (4.28) and (2.9), we have that

$$
\eta\left(\left\{u_{n}(t)\right\}_{n}\right)=0, \forall t \in J .
$$

Moreover, the set $\left\{f_{n}\right\}_{n}$ is semicompact since it is integrably bounded and $\eta\left(\left\{f_{n}(t)\right\}_{n}\right)=0$, for a.e. $t \in J$ (see (4.25) and (4.29)). Therefore, recalling again that $G_{S}$ satisfies properties $\left(G_{S} 1\right)$ and $\left(G_{S} 2\right)$, we can apply Theorem 5.1.1 of [10] so that the set $\left\{G_{S} f_{n}\right\}_{n}$ is relatively compact in $C(J ; X)$. Clearly, if a subset of $C(J ; X)$ is relatively compact, then its elements constitute an equicontinuous family on $J$. Hence, fixed $\varepsilon>0$, there exists $\delta_{1}(\varepsilon)=\delta_{1}\left(\frac{\varepsilon}{3}\right)>0$ such that for every $t_{1}, t_{2} \in J,\left|t_{1}-t_{2}\right|<\delta_{1}(\varepsilon)$ we have

$$
\left\|G_{S} f_{n}\left(t_{2}\right)-G_{S} f_{n}\left(t_{1}\right)\right\|<\frac{\varepsilon}{3}, \forall n \in \mathbb{N} .
$$


In addition, put $\delta_{2}(\varepsilon)=: \frac{\varepsilon}{3 Q \max \left\{K^{*}, K_{1}\right\}}$ we have (see $(p 4),(p 5),(2.7)$ and $\left.(g h 2)\right)$

$$
\begin{gathered}
\left\|C\left(t_{2}, 0\right) g\left(u_{n}\right)-C\left(t_{1}, 0\right) g\left(u_{n}\right)\right\| \leq Q \max \left\{K^{*}, K_{1}\right\}\left|t_{2}-t_{1}\right|<\frac{\varepsilon}{3}, \\
\left\|S\left(t_{2}, 0\right) h\left(u_{n}\right)-S\left(t_{1}, 0\right) h\left(u_{n}\right)\right\| \leq Q \max \left\{K^{*}, K_{1}\right\}\left|t_{2}-t_{1}\right|<\frac{\varepsilon}{3},
\end{gathered}
$$

for all $t_{1}, t_{2} \in J,\left|t_{2}-t_{1}\right|<\delta_{2}(\varepsilon), \forall n \in \mathbb{N}$.

Now, fixed $\delta(\varepsilon)=\min \left\{\delta_{1}(\varepsilon), \delta_{2}(\varepsilon)\right\}>0$, by (4.32), (4.33) and (4.34) we can deduce that, for every $t_{1}, t_{2} \in J$ such that $\left|t_{1}-t_{2}\right|<\delta(\varepsilon)$ we can say

$$
\begin{gathered}
\left\|y_{n}\left(t_{2}\right)-y_{n}\left(t_{1}\right)\right\| \leq\left\|C\left(t_{2}, 0\right) g\left(u_{n}\right)-C\left(t_{1}, 0\right) g\left(u_{n}\right)\right\|+ \\
+\left\|S\left(t_{2}, 0\right) h\left(u_{n}\right)-S\left(t_{1}, 0\right) h\left(u_{n}\right)\right\|+\left\|G_{S} f_{n}\left(t_{2}\right)-G_{S} f_{n}\left(t_{1}\right)\right\|<\varepsilon,
\end{gathered}
$$

for all $n \in \mathbb{N}$, i.e., the set $\left\{y_{n}\right\}_{n}$ is equicontinuous on $J$. So we conclude (see (2.10)):

$$
\lambda\left(\left\{y_{n}\right\}_{n}\right)=\bmod _{C}\left(\left\{y_{n}\right\}_{n}\right)=0
$$

From (4.20), by using (4.30) and (4.35) we deduce $\nu_{L}(\Omega)=0_{2}$.

Hence, condition $(I I)$ of $\nu_{L}$-condensity is verified too, therefore $\Gamma_{R}$ is $\nu_{L}$-condensing. Step 3: Finally we are in the position to apply Theorem 2.5. Hence the multioperator $\Gamma_{R}$ has a fixed point in $H_{R}$, i.e. there exists $x \in H_{R}$ such that

$$
x(t)=C(t, 0) g(x)+S(t, 0) h(x)+\int_{0}^{t} S(t, s) f(s) d s, t \in J
$$

where $f \in S_{F(., x(.))}^{1}$. Of course, $x$ is a mild solution for (2.6).

\section{An application}

Now we apply the result established in the preceding section to study the controllability of the following non-autonomous wave equation with initial conditions

$$
\left\{\begin{array}{l}
\frac{\partial^{2} w(t, \xi)}{\partial t^{2}}=\frac{\partial^{2} w(t, \xi)}{\partial \xi^{2}}+b(t) \frac{\partial w(t, \xi)}{\partial \xi}+f(t, w(t, \xi), u(t, \xi)), t \in J \\
w(t, 0)=w(t, 2 \pi), t \in J \\
\frac{\partial w(t, 0)}{\partial \xi}=\frac{\partial w(t, 2 \pi)}{\partial \xi}, t \in J \\
w(0, \xi)=\sum_{i=1}^{m} \frac{2 \pi t_{i}}{\xi_{i}}, \xi \in \mathbb{R} \\
\frac{\partial w(0, \xi)}{\partial t}=\sum_{i=1}^{m} \frac{2 \pi}{\xi}, \xi \in \mathbb{R} \\
u(t, \xi) \in U(t, w(t, \xi)), t \in J, \xi \in \mathbb{R}
\end{array}\right.
$$

where $0<t_{1}<\ldots<t_{i}<\ldots<t_{m}<a$ and $0<\xi_{1}<\ldots<\xi_{i}<\ldots<\xi_{m}<2 \pi$, $f: J \times \mathbb{C} \times \mathbb{C} \rightarrow \mathbb{R}, b: J \rightarrow \mathbb{R}$ and $U: J \times \mathbb{R} \rightarrow \mathcal{P}(\mathbb{R})$.

First, we fix the Banach space $X=L^{2}(\mathbb{T}, \mathbb{C})$, where $\mathbb{T}$ is the quotient group $\mathbb{T}=\mathbb{R} / 2 \pi \mathbb{Z}$ of all $2 \pi$-periodic 2 -integrable functions. As in [8], we will use the identification between the functions defined on $\mathbb{T}$ and the $2 \pi$-periodic functions from $\mathbb{R}$ to $\mathbb{C}$. Next, put the Sobolev space

$$
\begin{aligned}
H^{2}(\mathbb{T}, \mathbb{C})=\left\{X_{x}: \mathbb{T}\right. & \rightarrow \mathbb{C}: X_{x}([\xi])=x(\xi), x: \mathbb{R} \rightarrow \mathbb{C} \text { is } 2 \pi-\text { periodic }: \\
& \left.\exists x_{[0,2 \pi]}^{\prime}, x_{[0,2 \pi]}^{\prime \prime} \in L^{2}([0,2 \pi], \mathbb{C})\right\},
\end{aligned}
$$


provided by the norm

$$
\left\|X_{x}\right\|_{H^{2}(\mathbb{T}, \mathbb{C})}=\|x\|_{L^{2}([0,2 \pi], \mathbb{C})}+\left\|x^{\prime}\right\|_{L^{2}([0,2 \pi], \mathbb{C})}+\left\|x^{\prime \prime}\right\|_{L^{2}([0,2 \pi], \mathbb{C})},
$$

we consider the operator $A_{0}: D\left(A_{0}\right)=H^{2}(\mathbb{T}, \mathbb{C}) \rightarrow L^{2}(\mathbb{T}, \mathbb{C})$ so defined

$$
A_{0} X_{x}=\frac{d^{2}}{d \xi} x, \quad X_{x} \in H^{2}(\mathbb{T}, \mathbb{C})
$$

which is the infinitesimal generator of a strongly continuous cosine family $\left\{C_{0}(t)\right\}_{t \in \mathbb{R}}$, where $C_{0}(t): L^{2}(\mathbb{T}, \mathbb{C}) \rightarrow L^{2}(\mathbb{T}, \mathbb{C}$ ), for every $t \in \mathbb{R}$ (see [8]). Moreover, we fix the function $P: J \rightarrow \mathcal{L}\left(H^{1}(\mathbb{T}, \mathbb{C}), L^{2}(\mathbb{T}, \mathbb{C})\right)$ defined in this way

$$
P(t) X_{x}=b(t) \frac{d X_{x}}{d \xi}, \quad t \in J, \quad X_{x} \in H^{1}(\mathbb{T}, \mathbb{C}) .
$$

where we assume that the function $b: J \rightarrow \mathbb{R}$ of $(5.1)$ is $C^{1}$ on $J$. Now we are in the position to define the family $\{A(t): t \in J\}$, where, for every $t \in J, A(t): H^{2}(\mathbb{T}, \mathbb{C}) \rightarrow$ $L^{2}(\mathbb{T}, \mathbb{C})$, is an operator so defined

$$
A(t)=A_{0}+P(t), \quad t \in J .
$$

In [7] Henriquez has proved that this family generates a fundamental system $\{S(t, s)\}_{t, s \in J}$, which is compact (see [8], Lemma 4.1).

On the function $f$ we assume that $\tilde{f}: J \times H^{2}(\mathbb{T}, \mathbb{C}) \times L^{2}(\mathbb{T}, \mathbb{C}) \rightarrow H^{2}(\mathbb{T}, \mathbb{C})$ so defined

$$
\tilde{f}(t, x, u)([\xi])=f(t, x(\xi), u(\xi)), t \in J, x \in H^{2}(\mathbb{T}, \mathbb{C}), u \in L^{2}(\mathbb{T}, \mathbb{C}),[\xi] \in \mathbb{T},
$$

satisfies the following properties:

$(f 1)$ for every $x \in H^{2}(\mathbb{T}, \mathbb{C}), \quad u \in L^{2}(\mathbb{T}, \mathbb{C}), \tilde{f}(., x, u)$ is B-measurable;

$(f 2)$ for a.e. $t \in J, \tilde{f}(t, .,$.$) is continuous;$

(f3) there exists $k \in L_{+}^{1}(J)$ :

$$
\left\|\tilde{f}\left(t, x_{1}, u\right)-\tilde{f}\left(t, x_{2}, u\right)\right\|_{H^{2}([0,2 \pi], \mathbb{C})} \leq k(t)\left\|x_{1}-x_{2}\right\|_{H^{2}([0,2 \pi], \mathbb{C})},
$$

for a.e. $t \in J, x_{1}, x_{2} \in H^{2}(\mathbb{T}, \mathbb{C}), u \in L^{2}(\mathbb{T}, \mathbb{C})$;

Moreover we require that the multimap $\tilde{U}: J \times H^{2}(\mathbb{T}, \mathbb{C}) \rightarrow \mathcal{P}\left(L^{2}(\mathbb{T}, \mathbb{C})\right)$ so defined

$$
\tilde{U}(t, x)([\xi])=U(t, x(\xi)), t \in J, x \in H^{2}(\mathbb{T}, \mathbb{C}),[\xi] \in \mathbb{T},
$$

satisfies the conditions

(U0) for every $t \in J, \quad x \in H^{2}(\mathbb{T}, \mathbb{C}), \tilde{U}(t, x)$ is compact;

(U1) for every $x \in H^{2}(\mathbb{T}, \mathbb{C}), \tilde{U}(., x)$ is measurable;

(U2) for a.e. $t \in J, \tilde{U}(t,$.$) is upper semicontinuous;$

(U3) $\tilde{U}$ is superpositionally measurable, i.e. for every measurable multimap $V$ : $H^{2}(\mathbb{T}, \mathbb{C}) \rightarrow \mathcal{P}_{k}\left(L^{2}(\mathbb{T}, \mathbb{C})\right)$ the multimap $\tilde{U}(., V()$.$) is measurable;$

(U4) $\tilde{f}(t, x, \tilde{U}(t, x))$ is convex, $t \in J, x \in H^{2}(\mathbb{T}, \mathbb{C})$;

(U5) there exists $\alpha \in L_{+}^{1}(J)$ such that

$$
\|\tilde{f}(t, x, \tilde{U}(t, x))\|_{L^{2}([0,2 \pi], \mathbb{C})} \leq \alpha(t)\left(1+\|x\|_{H^{2}([0,2 \pi], \mathbb{C})}\right),
$$

for a.e. $t \in J$, for all $x \in H^{2}(\mathbb{T}, \mathbb{C})$; 
(U6) for every $t \in J, x \in H^{2}(\mathbb{T}, \mathbb{C})$ and for any bounded $\Omega \subset H^{2}(\mathbb{T}, \mathbb{C})$, the set $\tilde{f}(t, x, \tilde{U}(t, \Omega))$ is compact in $H^{2}(\mathbb{T}, \mathbb{C})$;

Now we introduce the map $F: J \times H^{2}(\mathbb{T}, \mathbb{C}) \rightarrow \mathcal{P}\left(H^{2}(\mathbb{T}, \mathbb{C})\right)$ so defined

$$
F(t, x)=\tilde{f}(t, x, \tilde{U}(t, x)), \quad t \in J, x \in H^{2}(\mathbb{T}, \mathbb{C}),
$$

and the maps $g: C\left(J ; H^{2}(\mathbb{T}, \mathbb{C})\right) \rightarrow H^{2}(\mathbb{T}, \mathbb{C})$ and $h: C\left(J ; H^{2}(\mathbb{T}, \mathbb{C})\right) \rightarrow H^{2}(\mathbb{T}, \mathbb{C})$ respectively defined in the following way

$$
\begin{aligned}
& g(x)([\xi])=\sum_{i=0}^{m} \frac{2 \pi t_{i}}{\xi_{i}}, \quad[\xi] \in \mathbb{T}, x \in C\left(J ; H^{2}(\mathbb{T}, \mathbb{C})\right) ; \\
& h(x)([\xi])=\sum_{i=0}^{m} \frac{2 \pi}{\xi_{i}}, \quad[\xi] \in \mathbb{T}, \quad x \in C\left(J ; H^{2}(\mathbb{T}, \mathbb{C})\right) .
\end{aligned}
$$

The previous arguments lead to revise a function $w: J \times \mathbb{R} \rightarrow \mathbb{C}$ such that

$$
\begin{gathered}
w(t, .) 2 \pi-\text { periodic }, \quad t \in J \\
\left.w(t, .)\right|_{[0,2 \pi]} \in L^{2}([0,2 \pi], \mathbb{C}), \quad t \in J
\end{gathered}
$$

as $x: J \rightarrow L^{2}(\mathbb{T}, \mathbb{C})$ so defined

$$
x(t)([\xi])=w(t, \xi), \quad t \in J, \quad[\xi] \in \mathbb{T} .
$$

Hence we can rewrite problem (5.1) in the form

$$
\left\{\begin{array}{l}
x^{\prime \prime}(t) \in[x(t)]^{\prime \prime}+b(t)[x(t)]^{\prime}+F(t, x(t)), t \in J \\
x(t)([0])=x(t)([2 \pi]), \quad[x(t)]^{\prime}([0])=[x(t)]^{\prime}([2 \pi]), \quad t \in J \\
x(0)=g(x) \\
x^{\prime}(0)=h(x)
\end{array}\right.
$$

First, we note that the conditions $(U 4)$ and (U6) imply respectively that the multimap $F$ (see (5.6)) takes convex and compact values. Moreover, by $(U 1)$ we can say that, for every $x \in H^{2}(\mathbb{T}, \mathbb{C})$, the multimap $Q_{x}: J \rightarrow \mathcal{P}\left(H^{2}(\mathbb{T}, \mathbb{C}) \times L^{2}(\mathbb{T}, \mathbb{C})\right)$ defined as $Q_{x}(t)=\{x\} \times \tilde{U}(t, x), t \in J$ is measurable. Hence from conditions $(f 1)$, $(f 2)$ we have that $F(., x)=\tilde{f}\left(., Q_{x}().\right)$ is measurable. Now by using the classical Kuratowski Ryll-Nardzewski measurable selection theorem we can conclude that the hypothesis $(F 1)$ is fulfilled. On the other hand, from $(U 2)$ we have that, for a.e. $t \in J$, the multimap $V_{t}: H^{2}(\mathbb{T}, \mathbb{C}) \rightarrow \mathcal{P}\left(H^{2}(\mathbb{T}, \mathbb{C}) \times L^{2}(\mathbb{T}, \mathbb{C})\right), V_{t}(x)=\{x\} \times \tilde{U}(t, x), x \in$ $H^{2}(\mathbb{T}, \mathbb{C})$, is upper semicontinuous and so, taking into account of $(f 2)$, Theorem 1.2.8 of [10] implies that $(F 2)$ holds. Moreover, by using $(U 5)$ we deduce that $F$ has the property $(F 3)$.

Next we prove that the multimap $F$ satisfies the condition $(F 4)$. Fixed $t \in J$ such that the property expressed in $(f 3)$ holds, we consider the multimap $B_{t}: H^{2}(\mathbb{T}, \mathbb{C}) \times$ $H^{2}(\mathbb{T}, \mathbb{C}) \rightarrow \mathcal{P}\left(H^{2}(\mathbb{T}, \mathbb{C})\right)$ defined as $B_{t}(x, y)=\tilde{f}(t, y, \tilde{U}(t, x)), \quad x, y \in H^{2}(\mathbb{T}, \mathbb{C})$. Then, we fix $x, y_{1}, y_{2} \in H^{2}(\mathbb{T}, \mathbb{C})$ and let $b_{1}=\tilde{f}\left(t, y_{1}, u\right)$ and $b_{2}=\tilde{f}\left(t, y_{2}, u\right) \in$ $B_{t}\left(x, y_{2}\right)$, where $u \in \tilde{U}(t, x)$. From $(f 3)$ there exists $k \in L_{+}^{1}(J)$ :

$$
\begin{gathered}
\left\|b_{2}-b_{1}\right\|_{H^{2}([0,2 \pi], \mathbb{C})}=\left\|\tilde{f}\left(t, y_{2}, u\right)-\tilde{f}\left(t, y_{1}, u\right)\right\|_{H^{2}([0,2 \pi], \mathbb{C})} \\
\leq k(t)\left\|y_{2}-y_{1}\right\|_{H^{2}([0,2 \pi], \mathbb{C})},
\end{gathered}
$$


by which we can deduce that the multimap $B_{t}(x,$.$) is k(t)$-Lipsichitz with respect to the Haudorff metric. Moreover we also can note that $(U 6)$ allows to say that, for every bounded subset $\Omega$ of $H^{2}(\mathbb{T}, \mathbb{C})$, the set $B_{t}(\Omega \times\{y\})=\tilde{f}(t, y, \tilde{U}(t, \Omega))$ is compact in $H^{2}(\mathbb{T}, \mathbb{C})$. Therefore all hypotheses of Proposition 2.2.2 of [10] are satisfied, hence we have

$$
\eta(F(t, \Omega))=\eta\left(\tilde{f}(t, \Omega \times \tilde{U}(t, \Omega))=\eta\left(B_{t}(\Omega \times \Omega)\right) \leq k(t) \eta(\Omega)\right.
$$

where $\eta$ is the Hausdorff MNC in $H^{2}(\mathbb{T}, \mathbb{C})$.

Then we can conclude that $(F 4)$ holds.

Finally, obviously the maps $g$ and $h$ have the properties $(g h 1)$ and $(g h 2)$ required in our existence theorem. Then from Theorem 4.1 we can deduce that there exists a continuous function $\hat{x}: J \rightarrow H^{2}(\mathbb{T}, \mathbb{C})$ that is a mild solution for (5.10), i.e.

$$
\hat{x}(t)=-\left.\frac{\partial}{\partial s} S(t, s)\right|_{s=0} g(u)+S(t, 0) h(u)+\int_{0}^{t} S(t, \xi) q(\xi) d \xi, \quad t \in J,
$$

where $q \in S_{F(., \hat{x}(.))}^{1}=\left\{p \in L^{1}(J ; X): p(t) \in F(t, \hat{x}(t))\right.$ a.e. $\left.t \in J\right\}$.

Now, since $\tilde{U}$ is superpositionally B-measurable (see (U3)), the multimap $Q: J \rightarrow$ $\mathcal{P}\left(H^{2}(\mathbb{T}, \mathbb{C}) \times L^{2}(\mathbb{T}, \mathbb{C})\right)$ so defined $Q(t)=\{(\hat{x}(t), \tilde{U}(t, \hat{x}(t))\}, t \in J$, having compact values (see $(U 0)$ ), is strongly measurable. Moreover, we recall that the multimap $F$ takes compact values in $H^{2}(\mathbb{T}, \mathbb{C})$ and that it has the properties $(F 1)$ and $(F 2)$. Hence, we are in the position to apply the Filippov implicit function lemma in the version furnished in ([15], Corollary 1.15). Then we can say that there exists a Bochner-measurable selection $\hat{u}: J \rightarrow L^{2}(\mathbb{T}, \mathbb{C})$ of the multimap $F(., Q())=.\tilde{f}(., \hat{x}(),. U(t, \hat{x}())$.$) . At this point, by considering the following func-$ tions $w: J \times \mathbb{R} \rightarrow \mathbb{C}$ and $u: J \times \mathbb{R} \rightarrow \mathbb{C}$ so defined

$$
\begin{aligned}
& w(t, \xi)=\hat{x}(t)([\xi]), \quad t \in J, \quad \xi \in \mathbb{R} \\
& u(t, \xi)=\hat{u}(t)([\xi]), \quad t \in J, \quad \xi \in \mathbb{R},
\end{aligned}
$$

we can conclude that $\{w, u\}$ is an admissible mild-pair for the problem (5.1).

Acknowledgements. T. Cardinali has contributed to this paper under the project GNAMPA "Differential Equations and Dynamical systems" of the Istituto Nazionale di Alta Matematica (INdAM) and under the project "Fondi Ricerca di Base 2016" (Department of Mathematics and Computer Science, University of Perugia).

\section{References}

[1] Ambrosetti, A., Un teorema di esistenza per le equazioni differenziali negli spazi di Banach, Rend. Sem. Univ. Padova, 39(1967), 349-360.

[2] Bungardi, S., Cardinali, T., Rubbioni, P., Semilinear nonlocal integro-differential inclusions via vectorial measures of noncompactness, In press on Appl. Anal. (2016), http://dx.doi.org/10.1080/00036811.2016.1227969.

[3] Couchouron, J.F., Kamenski, M., An abstract topological point of view averaging principle in the theory of differential inclusions, Nonlinear Analysis, 42(2000), 1101-1129.

[4] Cardinali, T., Rubbioni, P., On the existence of mild solutions of semilinear evolution differential inclusions, J. Math. Anal. Appl., 308(2005), no. 2, 620-635. 
[5] Denkowski, Z., Migorski, S., Papageorgiou, N.S., An Introduction to Nonlinear Analysis, Theory, Kluwer Acad. Publ. Boston/ Dordrecht/ London, 2003.

[6] Fattorini, H.O., Second Order Linear Differential Equations in Banach Spaces, NorthHolland Publishing Co., Amsterdam, 1985.

[7] Henríquez, H.R., Existence of solutions of non-autonomous second order differential equations with infinite delay, Nonlinear Anal., 74(10)(2011), 3333-3352.

[8] Henríquez, H.R., Poblete, V., Pozo, J.C., Mild solutions of nonautonomous second order problems with nonlocal initial conditions, J. Math. Anal. Appl., 412(2014), 1064-1083.

[9] Kozak, M., A fundamental solution of a second-order differential equation in Banach space, Univ. Iagel. Acta Math., 32( 1995), 275-289.

[10] Kamenskii, M., Obukhovskii, V., Zecca, P., Condensing Multivalued Maps and Semilinear Differential Inclusions in Banach Spaces, De Gruyter Ser. Nonlinear Anal. Appl. 7, Walter de Gruyter, Berlin, 2001.

[11] Kisynski, J., On cosine operator functions and one-parameter groups of operators, Studia Math., 44(1972), 93-105.

[12] Serizawa, H., Watanabe, M., Time-dependent perturbation for cosine families in Banach spaces, J. Math. 12, Houston, (1986), 579-586.

[13] Travis, C.C., Webb, G.F., Second order differential equations in Banach space, Nonlinear Equations in Abstract Spaces, Academic Press, New York, 1978.

[14] Vasilev, V.V., Piskarev, S.I., Differential equations in Banach spaces, II, Theory of cosine operator functions, J. Math. Sci. (N.Y.), 122(2004), 7047-7060.

[15] Zvyagin, V., Obukhovskii, V., Zvyagin, A., On inclusions with multivalued operators and their applications to some optimization problem, J. Fixed Point Theory Appl., 16(2014), $27-82$.

Tiziana Cardinali

Perugia University

Department of Mathematics and Computer Sciences

1, via Vanvitelli, 06123 Perugia, Italy

e-mail: tiziana.cardinali@unipg.it

Serena Gentili

Perugia University

Department of Mathematics and Computer Sciences

1, via Vanvitelli, 06123 Perugia, Italy

e-mail: gentilisere@hotmail.it 